\title{
Anabases
}

ANABASES Traditions et réceptions de l'Antiquité

19 | 2014

Varia

\section{Edme Béguillet traducteur de Martianus Capella? Enquête sur la réception des Noces de Philologie et de Mercure en France dans les années 1770-1780}

Jean-Baptiste Guillaumin

\section{(2) OpenEdition \\ Journals}

Édition électronique

URL : http://journals.openedition.org/anabases/4589

DOI : $10.4000 /$ anabases.4589

ISSN : 2256-9421

Éditeur

E.R.A.S.M.E.

Édition imprimée

Date de publication : 1 avril 2014

Pagination : 155-169

ISSN : $1774-4296$

\section{Référence électronique}

Jean-Baptiste Guillaumin, «Edme Béguillet traducteur de Martianus Capella ? Enquête sur la réception des Noces de Philologie et de Mercure en France dans les années 1770-1780 », Anabases [En ligne], 19| 2014, mis en ligne le 01 avril 2017, consulté le 21 octobre 2019. URL : http://journals.openedition.org/ anabases/4589; DOI : 10.4000/anabases.4589 


\section{Edme Béguillet traducteur de Martianus Capella? Enquête sur la réception des Noces de Philologie et de Mercure en France dans les années 1770-1780}

Jean-Baptiste Guillaumin

Composée au début du Ve S. AP. J.-C., l'œuvre de Martianus Capella connut une large diffusion au Moyen Âge et à la Renaissance. De fait, en insérant, au sein d'un prosimètre narrant le mariage allégorique du dieu Mercure avec la mortelle Philologie (l. I-II), sept exposés scientifiques attribués aux jeunes filles représentant les arts libéraux (Grammaire, Dialectique, Rhétorique, Géométrie, Arithmétique, Astronomie, Harmonie, 1. III-IX), les Noces de Philologie et de Mercure fournissaient plusieurs clefs de lecture susceptibles d'entrer en résonance avec les attentes intellectuelles des différentes époques : synthèse didactique des sciences antiques, récit bigarré relevant du genre de la satire ménippée, ou encore réflexion symbolique néoplatonicienne sur l'ascension par la connaissance. Les premières éditions semblent encore témoigner d'une réception relativement large du texte : le siècle qui sépare l'editio princeps $^{1}$ (1499) de

1 F. Bodianus, Opus Martiani Capelle de nuptiis Philologie et Mercurii libri duo ; De grammatica, de dialectica, de rhetorica, de geometri [sic], de arithmetica, de astronomia, de musica libri septem, cura Francisci Vitalis Bodiani, Vicenza. La date généralement retenue est 1499, mais on trouve parfois 1498 (voir par exemple M. Cappuyns, art. "Capella (Martianus) ", dans le Dictionnaire d'Histoire et de Géographie Ecclésiastiques 11, Paris, 1949, col. 835-848 - ici, 844 -, ou, plus récemment, la notice du SUDOC, http://www. sudoc.fr/098224840, consultée le 30 avril 2013) en raison d'une ambiguité dans la formulation : Anno Salutis MCCCCXCIX XVII Kalendas Ianuarias. 
celle de Grotius ${ }^{2}$ (1599) a ainsi laissé, au total, sept éditions ou réimpressions intégrales de l'œuvre de Martianus ainsi que quatre éditions partielles ${ }^{3}$ et une traduction italienne des deux premiers livres ${ }^{4}$. La diffusion de l'édition de Grotius, qui devient rapidement la référence, paraît avoir contribué au maintien d'un certain engouement pour Martianus Capella au XVII ${ }^{\mathrm{e}}$ siècle, dont témoignent également, dans des domaines très différents, la traduction italienne des deux premiers livres par Francesco Pona ${ }^{5}$, l'édition du livre IX (sur la musique) par H. Meibom au sein d'un corpus qui fit date dans l'histoire de la musicologie ${ }^{6}$, ou encore l'intérêt passager d'un Leibniz, qui avait le projet de se charger de l'édition de ce texte pour la collection Ad usum Delphini ${ }^{7}$.

En comparaison, le XVIII e s. apparait donc comme une sorte de temps faible, voire de "long purgatoire ${ }^{8}$ " dans la réception de Martianus Capella, avant une reprise

2 H. Grotius, Martiani Minei Felicis Capellae... Satyricon, in quo De nuptiis Philologiae \& Mercurii libri duo, \& De septem artibus liberalibus libri singulares, Leyde, C. Rapheleng, 1599. Comme dans toutes les éditions depuis l'editio princeps, le choix du titre suggère une distinction (qui n'est pas présente dans la tradition manuscrite, mais qu'on retrouvera fréquemment dans la réception de l'œuvre) entre le récit allégorique - livres I et II - et les exposés scientifiques - livres III à IX.

3 Voir sur ce point la liste présentée par J.-Y. Guillaumin, Martianus Capella. Les Noces de Philologie et de Mercure, Livre VII : l'Arithmétique, Paris, Les Belles Lettres, 2003, p. LXXXIX-XCIV. Sur les différentes éditions de l'œuvre de Martianus au XVI es., voir l'article de A. Moreno Hernández et M. Ayuso García, « La evolución de la concepción editorial de los primeros impresos incunables y postincunables de la obra de Marciano Capela (1499-1599)", Dialogues d'histoire ancienne 39/1 (2013), p. 121-175.

4 A. Buonacciuoli, Le nozze di Mercurio et di Filologia di Marziano Capella, tradotte dal latino dal Sig. Alfonso Buonacciuoli, nobile Ferrarese, Mantoue, Francesco Osanna, 1578.

5 F. Pona (sous le pseudonyme d'Eureta Misoscolo), Delle Nozze dell'Eloquenza con Mercurio, di Marziano Capella Cartaginese, libri due, tradotti da Eureta Misoscolo, Padoue, Gio. Battista Martini, 1629. Cette traduction et celle, plus ancienne, d'A. Buonacciuoli (cf. note ci-dessus) sont disponibles en fac-similé, accompagnées d'une étude introductive sur le contexte culturel et intellectuel de la réception italienne de Martianus à cette époque, dans l'ouvrage de G. Moretti, I primi volgarizzamenti italiani delle Nozze di Mercurio e Filologia, Trente, Università degli Studi di Trento, 1995.

6 М. Мегвом, Antiquae musicae auctores septem, Amsterdam, Elzevir, 1652.

7 C. Volpilhac-Auger (dir.), La collection Ad usum Delphini. L'Antiquité au miroir du Grand Siècle, Grenoble, ELLUG, 2000, en particulier p. 308-313 pour la correspondance de 1673 entre Leibniz et Huet.

8 Selon l'expression utilisée par C. VOLPILHAC-Auger, La collection..., p. 92 et développée note 106. On trouve toutefois, dans le domaine de la théorie musicale, quelques mentions de l'œuvre de Martianus au XVIII s. fondées sans doute sur l'édition du livre IX par M. Meiвom (cf. note 6) : c'est probablement cette édition qu'a utilisée J.-B. Dubois pour son développement sur la musique des Anciens dans les Réflexions critiques sur la poésie et sur la peinture (Paris, P.-J. Mariette, 1733), mais les auteurs qui s'inspirent de cette œuvre par la suite ne citent pas toujours le nom de Martianus Capella. De même, l'expression Musices seminarium accentus, tirée du livre des Noces consacré à la grammaire (III, 268), intéresse directement les théoriciens du rapport entre musique et texte au XVIII ${ }^{e}$ s., mais est généralement citée de 
des travaux philologiques sur cet auteur au XIX ${ }^{\mathrm{e}}$ siècle (la première édition moderne, agrémentée d'un commentaire détaillé, est celle de U.F. Kopp ${ }^{9}$, publiée en 1836). Si l'on recense, dans la seconde moitié du XVIII ${ }^{\mathrm{e}} \mathrm{s}$., trois éditions partielles (une édition du livre $\mathrm{V}$ dans un corpus de textes rhétoriques ${ }^{10}$ et deux éditions des deux premiers livres, correspondant au récit allégorique ${ }^{11}$ ), on ne connaît aucune édition complète, ni aucune traduction de cette encyclopédie allégorique romancée à l'époque, pourtant, de l'Encyclopédie et de l'engouement pour le roman ${ }^{12}$.

\section{Notes manuscrites d'Edme Béguillet}

Un document manuscrit, qui n'a guère été étudié, invite toutefois à mener une enquête plus approfondie sur la réception de Martianus Capella en France dans les années 1770-1780. Il s'agit de notes écrites par Edme Béguillet (1730-1786) dans les pages de garde de l'exemplaire de l'édition de Walthard qui se trouve à la $\mathrm{BnF}^{13}$ et provient du don fait par l'homme de lettres et bibliophile strasbourgeois Paul Ristelhuber à sa mort en $1899^{14}$. Dans ces notes, dont nous proposons ici une transcription complète,

manière floue ou erronée. Ainsi D. Diderot évoque, dans le Neveu de Rameau, «le passage suivant, que j’ai lu quelque part » (éd. J. Fabre, Genève, Droz, 1977, p. 78), et, dans le Salon de 1767 (éd. E.M. Bukdahl, M. Delon, A. Lorenceau, in Diderot, Euvres complètes, Paris, Hermann, 1990, t. XVI, p. 220), il l'introduit par l'expression « Je ne sais plus quel auteur a dit ", ce à quoi l'interlocuteur répond : "C'est Capella ".

9 U.F. Kopp, De nuptiis Philologiae et Mercurii et de septem artibus liberalibus libri novem, Francfort, F. Varrentrapp, 1836.

10 C. Capperonnier, Antiqui Rhetores Latini, e F. Pithoei bibliotheca olim editi (Rutilius Lupus, Aquila Romanus, Julius Rufinianus, de figuris Sententiarum et Elocutionis, etc. quibus nunc accedit M. Capellae de nuptiis Philologiae liber V), Strasbourg, 1756.

11 B.L. Walthard, Martianus Capella, editus cura L. Walthardi, Berne, Wagner, 1763 et J.A. Goez, Martiani Minei Felicis Capellae de Nuptiis Philologiae et Mercurii libri duo. Recensuit... et... illustravit Joann. Adam Goez, Nuremberg, Monath \& Kussler, 1794.

12 Sur cette absence manifeste des auteurs techniques et encyclopédiques latins chez les bibliophiles de cette époque, voir C. Volpilhac-Auger, "Comment peut-on être bibliophile ? Randon de Boisset, collectionneur et ami de Diderot ", D'une Antiquité l'autre. La littérature classique dans les bibliothèques $d u \mathrm{XV}^{e}$ au $\mathrm{XIX}^{e}$ s., Lyon, ENS éditions, p. 147-158, et en particulier p. 154.

13 Cote RES P-Z-545. Sur la deuxième de couverture est collée la notice de libraire suivante : " 11936. Capella (Martianus) editus cura L. Walthardi. Bernae, 1763, pet. in-8. v. m. 4 fr. Exemplaire portant la signature et des notes autographes de Béguillet, historien Bourguignon qui nous apprend qu'il a traduit en français l'œuvre de Capella, particularité qui semble avoir été ignorée des biographes et des bibliographes. »

14 On trouve bien, sur la page de titre, l'estampille circulaire à l'encre rouge qui caractérise ce don, avec l'indication du numéro, 11661 (nous remercions les services de la BnF de nous avoir transmis, au sujet du don Ristelhuber, la notice de L. PORTES figurant dans la base informatique intitulée Dictionnaire des fonds spéciaux de la BnF). 
E. Béguillet affirme avoir traduit les Noces de Philologie et de Mercure (probablement les livres 1 et 2 uniquement, conformément au contenu de l'édition de Walthard); il ajoute même, sous le titre imprimé, la mention "Traduit par M. Béguillet avocat membre de plusieurs academies ", accompagnée de sa signature, qui correspond bien, de même que la graphie, à ce que l'on trouve dans d'autres documents conservés ${ }^{15}$. Voici la transcription de ces notes manuscrites ${ }^{16}$ :

«V. la traduction que j’ai faitte de ce Roman Philosophique pour etre inseree dans la Biblioteque des Romans.

Le Mariage de Mercure et de la Mythoto Philologie par Martianus Capella né en Afrique Grammairien du $5^{\mathrm{e}}$ siecle est un des plus pretieux morceaux de la Litterature ancienne. On peut consulter ce qu'en a dit le savant Grotius dans l'édition in $8^{\circ}$ qu'il en a donnée avec des notes a Leyde en 1599. Il faut aussi voir celle de Basle in fol. 1577 avec le commentaire de Vulcanius. Cette edition cy faite par Valthard chez Vagner a Berne en 1763 a ete conparée avec toutes les precedentes; ainsi c'est la plus correcte pour la purete du texte quoique fautive en quelques endroits. On y a joint a la fin les variantes de Bongars et la liste de toutes les editions de cet ouvrage.

L'analyse que $j$ 'en ai faite est moins une traduction qu'une imitation pour faire connoitre cette piece singuliere ou l'on trouve la preuve que cette foule de divinités dont les cieux la terre la mer et les enfers etoient peuples n'etoient que des emblemes. Il y a lieu de s'etonner que le Savant auteur du monde | Primitif(M. Court de Gebelin) aïant

15 Plusieurs lettres autographes d'E. BÉGUILLET permettent de vérifier cette signature : une lettre du 30 octobre 1756, relevant de ses activités de notaire, est conservée dans le ms. 1183 de la bibliothèque municipale de Dijon (fonds Baudot), f. 290. On trouve également plusieurs lettres, sur des sujets divers, dans le manuscrit du fonds Moreau $\mathrm{n}^{\circ} 323$ (f. $30 \mathrm{r}$ à $52 \mathrm{v}$, à la $\mathrm{BnF}$ ). On peut citer, parmi d'autres exemples, la signature d'une lettre du 23 septembre 1773, accompagnée de la mention " avocat et premier notaire de la province de Bourgogne, membre de l'institut de Bologne des académies de Florence Montpellier Marseille Metz Caen » (f. 52 v) ; ou encore la signature d'une lettre du 5 septembre 1776, accompagnée de la mention : "correspondant de l'acad. des inscrip. et b. 1 . de celle des sciences " (f. 34 r). Par ailleurs, membre de la loge parisienne "La Réunion des Étrangers " (qu'il contribue à fonder en 1784), E. BÉGUILLET apparaît à plusieurs reprises dans les archives conservées au fonds maçonnique de la BnF. Voir notamment la "Demande en constitution pour la loge de la Réunion des Étrangers à l'Orient de Paris. Registrée le 12 janvier 1784 [...], accordée le 11 février 1784 » $\left(\mathrm{FM}^{2}\right.$ 97), où l'on trouve les indications suivantes : "Edme Beguillet, avocat en la Cour, de l'academie $\mathrm{R}^{\text {le }}$ des Sciences et de plusieurs academies, né le 13 octobre 1730, Secretaire $\mathrm{G}^{\mathrm{al}}$ a la correspondance et Garde des archives. Adresse : Rue St Jacques, Maison de M. Morin imprimeur ", avec une signature identique.

16 Nous conservons l'orthographe et la ponctuation d'origine (en normalisant toutefois l'utilisation des apostrophes). Même s'il ne s'agit pas d'une transcription diplomatique, nous indiquons les changements de pages par une barre verticale : le texte occupe en effet trois pages (verso du premier feuillet de garde, recto et une partie du verso du second). Enfin, nous transcrivons en italique les expressions soulignées dans les notes manuscrites. 
entrepris de prouver que la Theogonie et toutes les fables mythologiques des anciens n'etoient qu'une suite d'allegories physiques et morales ne se soit pas prevalu de l'ouvrage de Martianus Capella ou il auroit trouve la preuve complette de cette singuliere assertion qui est en meme tems lumineuse pour faciliter l'intelligence des anciens auteurs.

J'ai laisse quelques lacunes dans la traduction que j'ai donnée parce que je ne voulois pas interrompre la contexture de la fable. Ce seroit un service essentiel a rendre a la litterature francoise que de donner une traduction exacte et scrupuleuse d'un des plus curieux morceaux de l'antiquite payenne et de l'orner d'un savant commentaire dont eetlecy elle ne pourroit absolument se passer si l'on veut en avoir une parfaite intelligence.

Il sera difficile sans doute de reussir dans cette entreprise a moins que le traducteur ne soit en meme tems poete, philosophe, astronome, I physicien, mathematicien et litterateur universel. Mais ou trouver un pareil homme?"

Le deuxième paragraphe de cette note fournit quelques éléments biographiques très succincts sur Martianus Capella, puis, surtout, une synthèse bibliographique portant sur certaines éditions disponibles dans la seconde moitié du XVIII ${ }^{\mathrm{e}}$ s. : celle de H. Grotius, évidemment, qui est restée pendant plus de deux siècles l'édition de référence $^{17}$ (jusqu'à celle de U.F. Kopp en 1836), mais aussi celle de B. de Smet (dit Vulcanius), antérieure à celle de H. Grotius ${ }^{18}$. Quant à l'édition des livres I et II par Beat Ludwig Walthard, présentée comme « la plus correcte », elle correspond justement au volume sur lequel figurent les notes d'E. Béguillet : on peut vérifier la présence, à la fin du livre, à la fois des "variantes de Bongars ${ }^{19}$ " (p. 79-92), et de la « liste de toutes les éditions de cet ouvrage " (p. 93).

17 Voir ci-dessus, note 2. La présentation de l'édition de Grotius dans la liste de toutes les éditions de Martianus figurant à la p. 93 de ce volume (qu'E. BÉGuillet évoque à la fin du paragraphe) témoigne de son importance fondamentale, encore à cette époque : "praestantissima hactenus prodiit Editio Hugonis Grotii, ad cujus exemplar \& nostra fuit excusa ".

18 Bonaventura Vulcanius (B. De SMet), Isidori Hispalensis Episcopi Originum libri viginti ex antiquitate eruti. Et Martiani Capellae de nuptiis Philologiae et Mercurii libri novem..., Bâle, P. Perna, 1577. Voir sur cette édition l'article de A. Moreno Hernández et M. Ayuso García cité ci-dessus (note 3), p. 156.

19 Il s'agit des variantes proposées par l'érudit Jacques de Bongars dans les marges d'un exemplaire de l'édition de H. Petri (Bâle, 1532) conservé à la bibliothèque de Berne, comme on peut le déduire à la fois du titre de la section comportant les variantes («In M. Capellae de Philologia Libros II. Jac. Bongarsii et aliorum variae lectiones. ex Bibl. Bern. », p. 79) et de la mention présentant l'édition de H. Petri ("Margini hujus Editionis adscripsit variantes clar. Bongarsius, quas calci nostrae Editionis adjecimus », p. 93). Au total, une vingtaine de variantes attribuées à Bongars figurent dans la liste (avec la mention « Bong. » ou « Bongars. »). 


\section{Éléments biographiques et bibliographiques}

Il peut paraitre de prime abord étonnant que ce notaire dijonnais, que l'on connait essentiellement pour ses travaux d'agronomie et d'histoire de la Bourgogne ainsi que pour sa collaboration à l'Encyclopédie ${ }^{20}$, se soit intéressé à l'œuvre de Martianus Capella. Faut-il supposer que cet intérêt est lié à l'appartenance d'E. Béguillet à "plusieurs académies ", comme il l'écrit lui-même sur la page de titre ainsi que sous sa signature dans plusieurs lettres conservées ${ }^{21}$ ? De fait, il serait sans doute erroné

20 La liste de ses ouvrages imprimés figure chez J.-M. QUÉRARD, La France littéraire, Paris, Firmin Didot, 1827, t. 1, p. 255-256, puis chez P. M. Conlon, Le siècle des Lumières, Bibliographie chronologique, t. XXX, Index des auteurs A-E, 1761-1789, Genève, Droz, 2009, p. 78-79. On y trouve les titres suivants (nous restituons l'ordre chronologique) : De principiis vegetationis et agriculturae (1768), Mémoires sur les avantages de la mouture économique (1769), Enologie (1770), Dissertation sur l'ergot ou bled cornu (1771), Dissertation sur les essais et expériences (1771), Précis analytique du premier volume de l'histoire de Bourgogne (1771), Histoire des guerres des deux Bourgognes (1772), Traité de la connoissance générale des grains (1772), Traité de la mouture par économie (1772), Traité des subsistances (1772), Description générale et particulière du duché de Bourgogne (1774), Discours sur la mouture économique (1775), Manuel du meunier et du charpentier des moulins (1775), Précis analytique du traité général des grains (1779), Description historique de Paris (1779), Description générale et particulière de la France (1781), Considérations générales sur l'éducation (1782), Traité général des substances et des graines (1782), Traité pratique de la conservation des grains (1783), Discours sur l'origine, les progrès et les révolutions de la F $\therefore M \therefore$ philosophique (1784), Voyage pittoresque de la France (1784), Mémoire sur les moyens de perfectionner les moulins (éd. César Bucquet, 1786), Observations intéressantes et amusantes (éd. César Bucquet, 1783). Sur l'auteur lui-même, on consultera K.H. Doig, "Notices sur les auteurs des quatre volumes de "discours" du Supplément à l'Encyclopédie ", Recherches sur Diderot et l'Encyclopédie 9 (octobre 1990), p. 157-170 (la notice sur E. BÉGUILLET figure aux p. 158-159, avec quelques indications bibliographiques). Enfin, l'article le plus récent et sans doute le plus complet sur E. Béguillet est celui de P.-Y. Beaurepaire, "Dans l'ombre de l'abbé Courtépée. Edme Béguillet entre érudition provinciale et participation aux débats publics à la fin du XVIII siècle ", in D. Le Page, J. Loiseau, A. Rauwel (dir.), Urbanités : vivre, survivre, se divertir dans les villes ( $X V^{e}-X X^{e}$ s.) - Mélanges C. Lamarre, Dijon, Éditions Universitaires, 2012, p. 385-397.

21 Sur les relations entre E. BÉGuillet et l'Académie de Dijon (en lien avec la parution de certains de ses ouvrages), voir la thèse de R. Tisserand, Au temps de l'encyclopédie, L'Académie de Dijon de 1740 à 1793, Vesoul, Imprimerie nouvelle, 1936, p. 202-206. L'appartenance d'E. BÉGUILLET à plusieurs autres académies - et en particulier son statut de correspondant de l'Académie des Inscriptions et Belles Lettres - apparaît explicitement dans certaines de ses lettres (cf. ci-dessus, note 15), ainsi que dans le catalogue de ses ouvrages, mis en vente le 10 juillet 1786, après sa mort, qui s'intitule Notice des principaux articles de la bibliothèque de feu M. Edme Beguillet, Ancien Notaire des Etats de Bourgogne, Correspondant de l'Académie des Sciences, de l'Académie des Belles-Lettres de Paris, \& de l'Académie de Dijon (Paris, Morin). On trouve également une allusion ironique à cette 
de réduire les intérêts intellectuels du personnage aux seuls aspects agronomiques et historiques qui correspondent à son œuvre publiée : on sait en effet par un numéro du Mercure de France qu'il a entretenu une correspondance avec J.-P. Rameau au sujet de la musique des anciens ${ }^{22}$, et une lettre du 5 septembre 1776 , conservée dans le manuscrit $\mathrm{n}^{\circ} 323$ du fonds Moreau de la $\mathrm{BnF}$, évoque des "entreprises littéraires " auxquelles E. Béguillet ne put donner suite faute d'argent pour la publication ${ }^{23}$. Le catalogue du libraire Benoît Morin ${ }^{24}$, daté de 1783, annonce par ailleurs, du même E. Béguillet, un Choix des meilleures Pièces du Théâtre Italien moderne, présenté comme étant "sous presse " : l'ouvrage a bien été publié, mais son attribution à " M. E. B. D » (pour "Monsieur Edme Béguillet Dijonnais ») l'a longtemps fait échapper aux listes

accumulation de titres dans un dialogue satirique inédit rédigé par l'abbé C. BoullemieR et intitulé Entrevuë d'Edme Beguillet et de Philibert de la Mare aux Champs-Élisées (Dijon, BM, ms. 940 = fonds Baudot, f. 4-9) ; alors que Philibert de la Mare, rencontré aux Champs-Élysées, vient de déclarer : «je ne suis plus etonné [...] que pour ne point irriter l'envie, vous vous soyés contenté d'indiquer par des \&c les titres académiques dont vous avés été décoré », Edme BéGuillet lui répond : "Il est vrai que toutes les Académies non seulement du Royaume, mais des pais etrangers, sont venuës au devant de moi, et se sont fait honneur de me compter parmi leurs membres. "

22 Publication d'une lettre de J.-P. Rameau à E. BÉGuillet dans le Mercure de France, octobre 1765, p. 43-46, un an après la mort du compositeur. Pour introduire cette lettre, E. BÉGUILLET écrit (p. 43) : «Il seroit trop long de donner l'histoire de la dispute qui était entre M. Rameau et moi au sujet des effets de la Musique des anciens, et sur son ouvrage intitulé l'origine des Sciences. Le goût de la littérature ancienne n'est pas le dominant ; d'ailleurs les occupations de mon état et le respect que j'ai pour le public, ne me permettent pas de vous envoyer une dissertation informe qui a occasionné les deux lettres que M. Rameau m'a écrites sur cette matière. Celle dont je vous envoie copie est d'autant plus intéressante qu'elle rend raison du système musical de cet homme fameux : c'en est pour ainsi dire la clef ".

23 Ms. Moreau 323, f. 33v : «Si j'avois eu le bonheur de trouver à Paris une miserable place d'un millier d'ecus j'aurois pu donner suitte a mes entreprises litteraires mais tout cela sera perdu par defaut de moyens et d'occasions favorables. Ma femme fait des papillotes de mes recueils ". Cet intérêt pour des sujets multiples se trouve ensuite illustré par l'image du papillon (f. 34r) : " Je possede beaucoup de richesses litteraires mais je n'en retireray jamais le même fruit que Dom Villevieille avec de simples promesses. Denué de credit de protection et de moyens je n'ay plus de plans fixes dans mes etudes. Je voltige comme l'abeille de fleurs en fleurs ou plutot comme le papillon pour mon simple amusement, en attendant que le hazard me procure de l'employ ".

24 Catalogue des livres qui se trouvent chez Benồt Morin, Imprimeur-Libraire à Paris, rue S. Jacques, près celle de la Parcheminerie, à la Vérité, 1783 ; la dernière rubrique du fascicule présente les «Ouvrages de M. Beguillet, membre de plusieurs Académies, qui se trouvent chez le même Libraire ", le no XII étant précisément ce "Choix des meilleures Pieces du Théâtre Italien moderne, traduites en françois, avec des dissertations \& des notes, in- $8^{\circ}$, sous presse". 
bibliographiques ${ }^{25}$. Si l'on ajoute, à ce goût manifeste d'E. Béguillet pour des sujets multiples, son insistance sur sa bonne connaissance du latin ${ }^{26}$, on saisit mieux les raisons d'éventuels travaux de nature philologique. On notera tout particulièrement, dans le "catalogue des ouvrages imprimés et manuscrits de M. Béguillet ", publié en annexe de l'un de ses livres ${ }^{27}$, la mention d'une "Traduction de Lucrèce, avec des Notes » figurant dans la liste des ouvrages manuscrits $\left(n^{\circ} 13\right)$, parmi d'autres titres qui ne semblent guère mentionnés par les bibliographes - on ne relève toutefois aucune mention de Martianus Capella dans cette liste datant de 1775.

On trouve par ailleurs, dans son Discours sur l'origine, les progrès et les révolutions de la $F \therefore M \therefore$ philosophique (prononcé et publié en 1784) des considérations qui témoignent de son intérêt pour la philosophie, et en particulier pour la philosophie antique, notamment pythagoricienne. Ce discours, présenté comme une partie d'un travail plus large dont E. Béguillet annonce la publication prochaine (Histoire philosophique de l'Art Royal), s'attache à défendre le projet, proposé à l'origine par le propriétaire de la Bibliothèque des Romans, d'une souscription destinée à édifier un monument à Descartes $^{28}$. Dans ce discours, dont l'intérêt pour l'histoire de la franc-maçonnerie

25 La première étude précise de cet ouvrage se trouve dans un article récent de L. Giari, "Le pari du "choix des meilleures pièces du théâtre italien moderne" et le difficile rôle du répertoire italien à Paris ", in C.M. CEDERnA (dir.), Le théatre italien en France à l'époque des Lumières : entre attraction et dénégation, Lille, université de Lille III, 2012, p. 53-69. Dans le "Discours préliminaire " de cet ouvrage (p. VI), cité par L. Giari (p. 57), E. BÉGUILLET présente son projet en des termes très proches des notes manuscrites sur Martianus Capella : "On a cru que ce seroit rendre un service important à la Littérature Françoise et aux Auteurs qui travaillent pour nos Théâtres, de publier une Traduction, ou une imitation, quelquefois une simple analyse des Comédies modernes, qui ont le plus de réputation en Italie."

26 Lettre du 23 septembre 1773, BnF, fonds Moreau 323, f. $52 \mathrm{v}$ : l'auteur envoie un exemplaire de son traité De principiis vegetationis et agriculturae pour prouver sa maîtrise du latin (dans le dialogue inédit cité plus haut, n. 21, l'abbé Boullemier se moque de l'utilisation du latin pour un traité "destiné aux gens de la campagne»). On peut citer en complément le titre d'un ouvrage d'E. BÉGUILLET présenté comme étant " sous presse " dans le catalogue de la librairie Morin cité ci-dessus (n. 24) : « $\mathrm{n}^{\circ} \mathrm{XI}-$ Cours de philosophie botanique, traduite du latin de Linné avec un commentaire, contenant l'Histoire naturelle du Regne végétal, in $-4^{\circ}$ ".

27 Manuel du meunier et du charpentier de moulins ou Abrégé classique du traité de la mouture par économie, Paris, Panckoucke et Delalain, 1775, annexe non paginée.

28 E. BÉGullete, Discours sur l'origine... ("Avertissement ") : "On rend compte, au commencement de ce Discours, de l'occasion et des motifs qui y ont donné lieu. La R $\therefore$ L $\therefore$ Saint-Nicolas de la Parfaite Egalité avoit invité les VV $\therefore$ des autres LL $\therefore$ à se rendre au local du Contrat Social, le Dimanche 18 avril 1784, pour y délibérer sur une Souscription proposée par le Propriétaire de la Bibliothèque des Romans, dont le tiers seroit consacré à l'Érection d'un Monument destiné à recevoir les cendres de Descartes : cette proposition n'ayant pas été acceptée, l'Auteur de ce Discours soutint et s'engagea de prouver, qu'en vertu de l'alliance qui avoit subsisté de tout temps entre la Philosophie et la F $\therefore$ M $\therefore$, 
française a fait l'objet d'études récentes ${ }^{29}$, on se limitera ici à relever un motif qui entre en résonance avec les notes manuscrites sur Martianus Capella. Il s'agit du mot " emblème ", qui apparaît comme un terme central de ce discours (on en trouve plus d'une dizaine d'occurrences) : E. Béguillet définit ainsi le franc-maçon comme "un philosophe-pratique qui, sous les emblêmes religieux, adoptés de tous les temps par la sagesse, \& même par la haute philosophie [...], construit sur des plans tracés par la nature \& la raison, l'édifice moral de ses connaissances» (p. 7). Plus loin (p. 11), il qualifie la Tour de Babel d' "emblême ", en justifiant ce terme par une remarque qui n'est pas sans intérêt pour notre propos : "Car dans l'Antiquité les faits eux-mêmes sont aussi des allégories. " Cet intérêt pour les " emblèmes " fait donc écho au développement sur les dieux antiques que l'on trouve dans le troisième paragraphe des notes reproduites ci-dessus et permet sans doute de mieux comprendre l'intérêt que E. Béguillet pouvait trouver dans l'analyse allégorique du récit mythologique des Noces de Philologie et de Mercure.

Loin de l'image simplificatrice - généralement répandue - d'un agronome féru d'histoire bourguignonne, E. Béguillet apparaît donc, à la lumière de ces quelques compléments, comme un esprit encyclopédique foisonnant versé dans la littérature et la réflexion philosophique ${ }^{30}$. Ces différents éléments biographiques et bibliographiques donnent donc au personnage une envergure plus importante que ce qui apparaît de prime abord lorsqu'on se contente d'énumérer ses œuvres publiées, et incitent à moins de scepticisme face à l'existence même d'une traduction manuscrite des deux premiers livres de Martianus Capella par E. Béguillet.

c'étoit aux FF $\therefore$ MM $\therefore$ qu'il appartenoit exclusivement d'acquitter la dette que la Nation avoit contractée envers le Père de la Philosophie ». La proposition de souscription, faite par le "propriétaire de la Bibliothèque des Romans ", se trouve notamment, datée du 18 mars 1784, dans le Journal politique de Bruxelles (supplément du Mercure de France) $\mathrm{n}^{\mathrm{o}}$ 13, 27 mars 1784, p. 180-183, ainsi que dans le Journal des Savants de mai 1784, p. 313-314.

29 Voir notamment C. PORSET, Les Philalèthes et les convents de Paris. Une politique de la folie, Paris, Champion, 1996, p. 528-529, S. Van Damme, Paris, capitale philosophique : de la Fronde à la Révolution, Paris, Odile Jacob, 2005, p. 226-227, ainsi que P.-Y. Beaurepaire, «Dans l'ombre de l'abbé Courtépée... » (cf. ci-dessus, note 20), p. 393-397.

30 On rejoint ici les conclusions des deux articles les plus récents consacrés à ce personnage : ainsi L. GIARI ("Le pari du "choix des meilleures pièces du théâtre italien moderne"... ", p. 56) écrit qu' " en lisant les œuvres de Béguillet et en particulier leurs préfaces, on découvre un intellectuel exemplaire du XVIII ${ }^{\mathrm{e}}$ s. " et P.-Y. BEAUREPAIRE conclut son étude ( Dans l'ombre de l'abbé Courtépée... », p. 397) en ces termes : «Edme Béguillet mérite donc d'intéresser les historiens, non seulement en raison de la richesse de son ouvre imprimée, mais aussi de sa volonté d'affirmer son expertise dans les domaines d'étude qu'il affectionne, et de sa prise de parole déterminée dans les débats qui animent alors l'espace public. En cela, il est vraiment un homme de son temps". 


\section{Une traduction pour la Bibliothèque des Romans?}

Quel a donc pu être le destin de cette traduction ? Il ne nous a pas été possible, pour l'instant, d'en retrouver la trace. Contrairement à ce que l'auteur indique, on ne relève rien de tel dans l'ensemble des volumes de la Bibliothèque universelle des romans, collection lancée en juillet 1775, sous la responsabilité d'A.-R. de Voyer d'Argenson, Marquis de Paulmy (associé à L.-É. de La Vergne, Comte de Tressan), remplacé après décembre 1778 par J.-F. de Bastide (propriétaire du privilège dès le lancement), et qui devait cesser de paraître en $1789^{31}$. Dans l'un des numéros, le " propriétaire de la Bibliothèque des Romans» invite "MM. les Gens-de-Lettres à lui procurer de nouvelles richesses ${ }^{32}$ " : il est donc tout à fait plausible qu'E. Béguillet ait rédigé une traduction dans le but de l'envoyer à la Bibliothèque des Romans. On peut par ailleurs rappeler que la souscription en l'honneur de Descartes, qui constitue le point de départ du Discours sur l'origine, les progrès et les révolutions de la $F \therefore M \therefore$ philosophique, fut précisément lancée par le "propriétaire de la Bibliothèque des Romans ${ }^{33}$ ", ce qui, sans prouver nécessairement la proximité entre les deux personnages, traduit au moins une communauté d'intérêts intellectuels en 1784. Or les travaux exhaustifs réalisés par A. Martin sur la Bibliothèque des Romans ne mentionnent jamais Martianus Capella, ni E. Béguillet. Une traduction ne retenant que le récit allégorique des Noces de Philologie et de Mercure aurait pourtant eu toute sa place dans une collection dont le prospectus de lancement ${ }^{34}$ affirme que "[les romans] sont comme autant de tableaux allégoriques qui présentent la vérité voilée ou embellie par la fiction » et qui, à ses débuts au moins, consacre la première "classe » de chaque numéro aux « romans traduits du grec et du latin ${ }^{35}$ ". On notera par ailleurs, dans le numéro de mai 1780, la remarque suivante (p. 184) : "Nous avons appris qu'il existoit une chaîne de Romans mythologiques, traités par un Homme de Lettres familiarisé avec toutes les fictions des Anciens [...]. Nous avons su

31 Sur les éditeurs et rédacteurs de la Bibliothèque des romans, voir R. POIRIER, La bibliothèque universelle des romans. Rédacteurs, textes, public, Genève, Droz, 1976, en particulier p. 9-31, ainsi que A. MARTin, La bibliothèque universelle des romans, 1775-1789, Présentation, table analytique et index, Oxford, The Voltaire Foundation, 1985, en particulier p. 5-21. On trouvera une utile synthèse sur cette collection dans la notice de K.H. Dolg pour l'"Édition électronique revue, corrigée et augmentée du Dictionnaire des journaux (1600-1789) ", disponible sur http://dictionnaire-journaux.gazettes18e.fr/ journal/0172-bibliotheque-universelle-des-romans (site consulté le 30 avril 2013).

32 Bibliothèque des Romans, avril 1780, p. 5.

33 Voir ci-dessus, note 28.

34 Publié dans plusieurs journaux, notamment le Mercure de France, mai 1775, p. 168, et inséré en tête du premier volume de la Bibliothèque universelle des romans, juillet 1775, p. 5.

35 On trouve cet intitulé de juillet 1775 à juin 1776 ; cette " classe » est ensuite intitulée «Romans grecs, latins, espagnols, etc. » (de juillet 1776 à novembre 1777), puis « Romans étrangers " (à partir de janvier 1778). 
qu'il se disposoit à les faire imprimer ", ce qui justifie l'ajout d'une nouvelle " classe " consacrée à des romans inspirés de la mythologie antique (le premier de ces romans s'intitule ainsi Amours de Glauque ou la vengeance de Vénus ${ }^{36}$ ). On constate également l'apparition, dans les numéros de décembre 1785 à février 1786, d'une nouvelle classe consacrée aux "romans allégoriques ${ }^{37}$ ". Or, malgré cet intérêt manifeste pour la traduction de romans antiques (dans les premiers numéros de la collection) puis pour la mythologie et pour l'allégorie, aucun titre de la Bibliothèque universelle des romans ne semble correspondre à une traduction des Noces de Philologie et de Mercure.

\section{Antoine Court de Gébelin lecteur de Martianus Capella}

Peu d'éléments permettent de préciser la datation des notes manuscrites d'E. Béguillet. On relève toutefois une allusion à l'ouvrage monumental d'Antoine Court de Gébelin, Le Monde primitif analysé et comparé avec le monde moderne, qui parut en neuf volumes de 1773 à la mort de l'auteur en 1784, et connut une diffusion relativement importante ${ }^{38}$. Or, contrairement à ce qu'affirme E. Béguillet, on trouve plusieurs mentions de Martianus Capella dès le premier volume de l'œuvre d'A. Court de Gébelin dans la deuxième partie, intitulée Allégories orientales ou le fragment de Sanchoniaton), dont le propos consiste précisément à ramener la mythologie ancienne à une série d'allégories. Martianus Capella est ainsi cité au sujet de l'étymologie du terme heros rapproché de "Hera, qui désigne la Terre " (p. $45=$ Nupt. 2, 160), au sujet de Proserpine appelée "Centésima" (p. $57=$ Nupt. $\left.1,81^{39}\right)$, au sujet des dieux égyptiens, correspondant aux planètes (p. $66=$ Nupt. $2,18^{40}$ ), au sujet de l'Ibis qui porte le nom d'un mois égyptien (p. 106 et $120=2,178$ ), et au sujet des dieux Azonoi, c'est-à-dire "sans zone » (p. 220

36 A. MARTIn, La bibliothèque universelle des romans..., p. 159, évoque un « texte donné pour manuscrit, qui semble être inspiré par les livres XIII-XIV des Métamorphoses d'Ovide ", qu'il ne parvient pas à identifier.

37 Cf. A. Martin, La bibliothèque universelle des romans..., p. 216-219. Les titres sont les suivants : Hégésippe ou l'ile de la médiocrité, conte allégorique (décembre 1785), La pudeur et L'amour converti - deux récits mythologiques - (janvier 1786), La naissance de Clinquant (février 1786).

38 Sur cet ouvrage, voir l'étude d'A.-M. Mercier-Faivre, Un supplément à L'Encyclopédie : Le Monde primitif d'Antoine Court de Gébelin, Paris, Champion, 1999.

39 A. Court de Gébelin situe la référence "p. 21 ", ce qui correspond à l'édition de H. Grotius (cf. ci-dessus, note 2), qui sert de référence encore à cette époque (malgré l'existence, pour les livres I et II, de l'édition de Walthard mentionnée au début de cette étude, cf. note 11). Selon l'usage, nous citons les passages de Martianus en mentionnant le numéro du livre, puis le paragraphe dans l'édition de U.F. Kopp (cf. ci-dessus, note 9).

40 Dans ce passage, A. CourT DE GÉBELIn propose même une traduction : " On voyoit dans un cercle Solaire un vaisseau avec sept Pilotes, qui étoient freres \& parfaitement semblables l'un à l'autre. Et ce vaisseau étoit rempli d'une lumiere céleste, intarissable, qui se répandoit dans tout l'Univers. » 
= Nupt. 1, 61). L'oubli dans lequel était tombé Martianus apparaît toutefois à travers la formulation des références bibliographiques faites par A. Court de Gébelin, qui évoque, dans la première référence citée ci-dessus (p. 45), " une étymologie que les Anciens avoient aperçue \& que l'on peut voir dans l'ouvrage d'un Proconsul romain cité en note " (il l'appelle ensuite tantôt « le Proconsul Capella », tantôt " Marcien Capella ») : ces flottements suffisent à indiquer que le nom de Martianus Capella ne devait guère être familier aux lecteurs. On retrouve également plusieurs références à Martianus dans le volume IV du Monde primitif, paru en 1776 et consacré au calendrier. Ces mentions concernent des sujets extrêmement variés : divinités appelées ou Lar Caelestis et Lar Cunctalis (p. $387=$ Nupt. 1, 48 et 1, 54) ; étymologie de Faunus (p. $409=$ Nupt. 2, 167) ; légende du rétablissement d'Osiris (p. 539-540 = Nupt. 2, 126 ${ }^{41}$ ). Surtout, on peut lire, au sein d'un développement sur l'identification du Soleil à Bacchus (p. 544-546), une traduction commentée de l'hymne adressé par Philologie au Soleil au livre II des Noces $\left(2,185-193^{42}\right)$; la présentation de cette traduction confirme l'oubli manifeste de l'œuvre de Martianus à cette époque : "Comme [l'hymne au Soleil] est peu connue, \& qu'elle renferme un précis de la Théologie Egyptienne \& Pythagoricienne à l'égard du Soleil, nous avons cru devoir la mettre sous les yeux de nos Lecteurs, en l'accompagnant d'un essai de traduction \& de quelques observations ». En raison de son intérêt pour l'étude de la réception de Martianus, nous citons le passage dans son intégralité (en conservant les graphies d'origine) :

«Force suprême du Père inconnu, son premier né, principe du sentiment $\&$ de l'intelligence, source de lumiere, regne de la nature, gloire des Dieux, preuve de leur existence, œil du Monde ; éclat de l'Olympe resplendissant, auquel seul il est permis de voir le Pere placé au-delà du Monde $\&$ de considérer le grand Dieu ; vous qui dans vos immenses tours gouvernez l'Univers \& ses révolutions : car vous en parcourez le milieu, donnant seul aux mondes supérieurs une chaleur tempérée, \& dictant vos loix aux Astres sacrés des Dieux, parce que vous êtes placé dans le quatriéme orbite ; \& que votre nombre vous a été assigné par la droite raison, ensorte que dès le commencement, vous nous donnez un double tetrachorde. Le Latium vous appelle solEIL, parce que seul vous êtes après le Pere, la source de la lumière. Douze rayons couronnent votre tête sacrée, parce que vous formez autant d'heures. Quatre Coursiers sont attelés à votre char, parce que seul vous domptez le quadrille formé par les Elémens. Comme en dissipant les ténébres, vous manifestez la

41 Les quatre vers cités (Qui fata succidentis / Separat libens Osiridis (sic) / Sationibus grauari / Genitalibus repertis) sont " paraphrasés " ainsi, p. 540 : "Osiris, par ses soins, délivré du Tombeau, / Recommence aussitôt sa course vagabonde, / Éclairant les Mortels de son divin flambeau, / Et couvrant de ses biens la Terre qu'il féconde ".

42 Sur les difficultés propres au texte de Martianus, on consultera en particulier L. LENAz, Martiani Capellae De nuptiis Philologiae et Mercurii liber secundus, Padoue, Liviana Editrice, 1975, p. 46-61 et 219, ainsi que L. Cristante, Martiani Capellae De Nuptiis Philologiae et Mercurii Libri I-II, Hildesheim, Weidmann, 2011, p. 331-338. 
lumiere des Cieux, on vous appelle PHÆBUS qui découvre les secrets de l'avenir ; \& LYÉUS, parce que vous dissipez les mystères de la nuit. Le Nil vous adore sous le nom de SERAPIS ; Memphis, sous celui d'osiris. Dans les Fêtes d'Hyver vous êtes appelé MITHRAS, PLUTON, le barbare TYPHON. On vous revére aussi sous les noms du bel ATYS, de l'ENFANT chéri de la charrue. Dans la brûlante Lybie, vous êtes AMMON ; et à Biblos, ADONIs. Ainsi l'Univers entier vous invoque sous des noms différens. Je vous salue, véritable face des Dieux, image de votre Pere, vous dont trois lettres qui valent en nombre six cent huit, forment le nom sacré, le surnom \& le présage. Accordez-nous, ô Pere, de monter dans les assemblées étherées de l'Esprit ; \& de contempler, à la faveur de votre nom sacré, le Ciel étincelant de flambeaux."

L'explication donnée ensuite (p. 546-548) utilise différents éléments de l'hymne pour démontrer l'identité entre Bacchus et le Soleil (sujet général du développement) : allusion à la place du soleil comme " mèse " dans l'harmonie des sphères - qui, selon l'auteur, constitue un écho au nom Míons rapproché de Bacchus dans l'hymne orphique 42 ; emploi des noms Lyéus et Adonis ${ }^{43}$, ainsi que de l'expression curui et puer almus aratri (" l'enfant chéri de la charrue » dans la traduction), qui évoquerait Iaccos $^{44}$; enfin, A. Court de Gébelin rattache le calcul arithmologique de la fin de l'hymne au nom 'Y $\tilde{\eta} \varsigma(\mathrm{Y}=400, \mathrm{H}=8, \Sigma=200)$, "l'humide ", en lien avec la constellation pluvieuse des Hyades, considérées comme les nourrices de Bacchus ${ }^{45}$.

C'est donc ce syncrétisme mythologique, interprété de manière allégorique, qui semble avoir particulièrement intéressé A. Court de Gébelin, dans un contexte général marqué par une redécouverte de la mythologie égyptienne et de sa portée symbolique $^{46}$. Il n'est à cet égard pas surprenant que toutes les références mentionnées se trou-

43 A. Court de GéBelin recourt à un passage de Plutarque (Quaest. conv. 671 B) et à l'hymne orphique 56 à Adonis pour assimiler Adonis et Bacchus.

44 "Ce fils chéri, c'est celui qu'on invoquoit dans les Mystères de Cérès sous le nom d'Iackus $\&$ qui étoit regardé comme le fils de Jupiter \& de Proserpine, ou de Cérès elle-même, selon d'autres. Là, il étoit représenté comme enfant, avec un van, emblème de l'Agriculture ", p. 547.

45 Jean Scot avait déjà proposé, au milieu du $\mathrm{IX}^{\mathrm{e}}$ s., d'analyser ce jeu arithmologique comme un écho à HY $\Sigma$ (éd. C.E. LuTz, Iohannis Scotti annotationes in Marcianum, Cambridge, Mass., The Mediaeval Academy of America, 1939, p. 72). L'autre hypothèse au sujet de cette énigme arithmologique (avancée au moins depuis l'édition de U.F. Kopp, cf. ci-des-

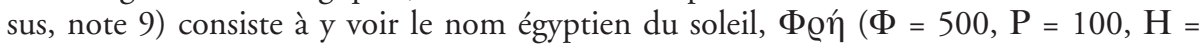
8). Le vers cité par A. COURT DE GÉBELIN (conformat sacrum nomen, cognomen et omen) correspond au texte de l'editio princeps (Venise, 1499), encore repris par H. Grotius, et non à celui des manuscrits, que retiennent les éditeurs postérieurs (conformat sacrum mentis, cognomen et omen). Les commentaires sur $\mathrm{HY} \Sigma$ et $\mathrm{YH} \Sigma$ s'inspirent de la note ad loc. de H. Grotius.

46 Cet engouement peut être illustré par l'ouvrage de P.E. Jablonski, Pantheon Aegyptiorum, sive de diis eorum commentarius, cum prolegomenis de religione et theologia Aegyptiorum, 3 vol., Francfort, 1750-1752, souvent cité par A. CourT DE GÉBELIN, et qui mentionne 
vent dans les livres I et II de Martianus, qui correspondent à la partie allégorique des Noces de Philologie et de Mercure - les exposés scientifiques des livres III à IX, considérés sans doute comme un état dépassé des connaissances, étant manifestement laissés de côté. Enfin, la différence, dans la manière de citer Martianus, entre le tome I (1773) et le tome IV (1776) paraît indiquer, de la part d'A. Court de Gébelin, une redécouverte des Noces (ou en tout cas de ses deux premiers livres) entre ces deux dates : si la lecture proposée en 1773 reste assez superficielle, présentant implicitement Martianus comme un auteur presque inconu, les remarques de 1776 attestent une lecture plus approfondie, allant jusqu'à la citation et à la traduction.

On constate donc un décalage entre l'utilisation de Martianus par A. Court de Gébelin et la remarque d'E. Béguillet, s'étonnant du fait que ce dernier " ne se soit pas prévalu de l'ouvrage de Martianus Capella ", ce qui est d'autant plus surprenant que l'on trouve, dans la "table des matières » du tome IV du Monde primitif, le renvoi suivant : "Marcien Capella, son hymne au Soleil, 544 »(p. 620). Faut-il en déduire qu'E. Béguillet n'eut qu'une lecture distraite de l'ouvrage d'A. Court de Gébelin, ou supposer que la note manuscrite date d'avant la parution du tome IV du Monde primitif, qui contient les allusions les plus nettes (en particulier la traduction complète de l'hymne au soleil) ? Cette dernière hypothèse (que rien ne permet toutefois de confirmer de manière irréfutable) donnerait un terminus ante quem en 1776-1777, en supposant que les nouveaux volumes du Monde primitif aient été largement diffusés et lus dès leur parution. La note manuscrite étudiée ici daterait alors de 1775-1776, puisqu'elle est nécessairement postérieure au premier numéro de la Bibliothèque universelle des romans ${ }^{47}$, et que la traduction mentionnée ne figure pas dans la liste des ouvrages imprimés et manuscrits de Béguillet ${ }^{48}$. Il s'agirait donc précisément de l'époque où E. Béguillet écrit qu'il "voltige comme l'abeille de fleurs en fleurs ou plutôt comme le papillon pour [son] simple amusement ${ }^{49}$ ". On peut imaginer que, par la suite, occupé par d'autres travaux ou rebuté par la difficulté de la tâche, il se soit détourné de son premier enthousiasme pour Martianus Capella et n'ait plus songé à reprendre sa traduction pour en assurer la publication. Quoi qu'il en soit, la confrontation des notes manuscrites d'E. Béguillet et des citations de Martianus chez A. Court de Gébelin témoigne d'une volonté de redécouvrir un auteur ardu dont la pleine compréhension nécessite, selon les termes d'E. Béguillet, « un traducteur qui soit en même temps poète, astronome, physicien, mathématicien et littérateur universel ».

lui-même Martianus dans quelques passages (cf. par exemple I, p. 108 et 255, ou encore V, 162-163, passage cité par A. COURT DE GÉBELIN au sujet de l'interprétation de l'Ibis comme mois égyptien par Martianus Capella).

47 Voir ci-dessus, note 31.

48 Voir ci-dessus, note 27.

49 Voir ci-dessus, note 23. 


\section{Conclusion}

Si ces quelques lignes manuscrites d'E. Béguillet conservent un aspect mystérieux dans la mesure où elles évoquent une traduction inconnue par ailleurs, elles nous ont toutefois incité à approfondir l'enquête sur la réception de Martianus Capella dans les années 1770-1780, durant lesquelles l'intérêt philosophique pour l'allégorie (dont témoignent l'œuvre de A. Court de Gébelin comme les remarques manuscrites d'E. Béguillet) vient compléter le goût de l'époque pour le roman mythologique (qui apparaît comme nouvelle classe dans la Bibliothèque universelle des Romans en mai 1780) : on relève ainsi un contexte favorable aux travaux sur Martianus et, en particulier, à une traduction des livres I et II, même si aucun travail de grande envergure n'est conservé. À travers les quelques lignes manuscrites éditées ci-dessus, E. Béguillet apparait donc comme un homme de lettres désireux de mettre au goût du jour des ouvres peu connues de ses contemporains ${ }^{50}$. À l'issue de cette étude, il ne fait guère de doute que la traduction de Martianus Capella par E. Béguillet a bien existé sous forme manuscrite : qu'une version ait été envoyée ou non à la Bibliothèque des Romans, il est plausible que ce texte ait fait partie du lot $\mathrm{n}^{\circ}$ LXVII vendu à la mort de l'auteur, et comprenant "plusieurs Manuscrits sur différens sujets ${ }^{51}$ ». Il reste à souhaiter que les hasards de la circulation des textes permettent un jour de découvrir cette traduction inédite.

Jean-Baptiste Guillaumin

Université Paris-Sorbonne
1, rue Victor Cousin,
75005 Paris
jean-baptiste.guillaumin@paris-sorbonne.fr

50 De même que la relative méconnaissance du théâtre italien a servi d'argument au Choix des meilleures pièces du théatre italien moderne, comme le montre L. GIARI dans l'article cité plus haut (note 25).

51 Notice des principaux articles de la bibliothèque de feu M. Edme Béguillet (voir ci-dessus, note 21), p. 15. 